\title{
An integrated approach to study genetics of Triticum aestivum vegetation period
}

\author{
Kiseleva A.A. ${ }^{1,2 *}$, Stasyuk A.I. ${ }^{1}$, Bragina M.K. ${ }^{1}$, Berezhnaya A.A. ${ }^{1,3}$, \\ Kolozhvari A.E. ${ }^{1,3}$, Leonova I.N. ${ }^{1}$, Kochetov A.V. ${ }^{1,2}$, Salina E.A. ${ }^{1,2}$ \\ ${ }^{1}$ Institute of Cytology and Genetics, SB RAS, Novosibirsk, Russia \\ ${ }^{2}$ Kurchatov Genomic Center of the Institute of Cytology and Genetics, SB RAS, Novosibirsk, Russia \\ ${ }^{3}$ Novosibirsk State University, Novosibirsk, Russia \\ * email: antkiseleva@bionet.nsc.ru
}

The vegetation period consists of two main stages - the time from seedling to heading and the time from heading to maturation. The key factors involved in the transition of a plant from the vegetative to the generative developmental phase are identified, however many components and mechanisms of this pathway are still unknown. Here, we aimed to study the different components and functioning of this path in detail. Methods of classic genetics included identification and characterization of novel alleles ( $\mathrm{Vrn}-\mathrm{B3})$ and study of the interaction between main flowering genes (Vrn-B3a, Ppd-D1, and Vrn1). Bioinformatics methods let us study wheat diurnal transcriptome to reveal components of circadian rhythms involved in the transition of plants to different developmental stages. Methods of genome editing with gRNA/Cas9 system are to study the regulation of expression of $P p d-D 1$ gene. This work is currently in progress and we guess it will result in plants with shortened flowering time. The genetics of the second part of the vegetation period (maturation time) is now poorly understood. In this work, we have shown that in the long day the vegetation period is most influenced by the period from heading to maturation, but not heading time itself. Methods of quantitative genetics (GWAS, QTL analysis) let us identify novel loci and dissect candidate genes for wheat maturation time. Thus, in this work we advanced our knowledge about genetic determinants of the first stage of the vegetation period (heading time) and identified new genetic factors influencing the second stage (maturation time).

Acknowledgements: This work was supported by the Kurchatov Genomic Center of the Institute of Cytology and Genetics, SB RAS (075-15-2019-1662). 